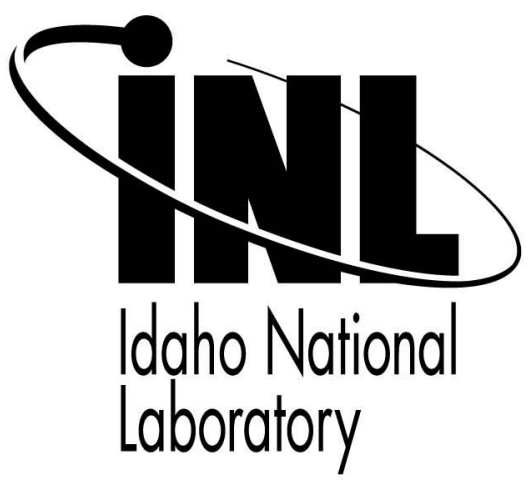

\title{
Surface Bonding Effects in Compound Semiconductors Nanoparticles: II
}

\section{Physics and Chemistry of Semiconductor Interfaces}

\author{
H. H. Farrell
}

July 2008

This is a preprint of a paper intended for publication in a journal or proceedings. Since changes may be made before publication, this preprint should not be cited or reproduced without permission of the author. This document was prepared as an account of work sponsored by an agency of the United States Government. Neither the United States Government nor any agency thereof, or any of their employees, makes any warranty, expressed or implied, or assumes any legal liability or responsibility for any third party's use, or the results of such use, of any information, apparatus, product or process disclosed in this report, or represents that its use by such third party would not infringe privately owned rights. The views expressed in this paper are not necessarily those of the United States Government or the sponsoring agency. 


\title{
Surface Bonding Effects in Compound Semiconductors Nanoparticles:
}

II

H. H. Farrell, Idaho National Laboratory, P.O. Box 1625, Idaho Falls, ID 83415-2211

*email: Helen.Farrell@inl.gov

\begin{abstract}
Small nanoparticles have a large proportion of their atoms either at or near the surface, and those in clusters are essentially all on the surface. As a consequence, the details of the surface structure are of paramount importance in governing the overall stability of the particle. Just as with bulk materials, factors that determine this stability include "bulk" structure, surface reconstruction, charge balance and hybridization, ionicity, strain, stoichiometry, and the presence of adsorbates. Needless to say, many of these factors, such as charge balance, hybridization and strain, are interdependent. These factors all contribute to the overall binding energy of clusters and small nanoparticles and play a role in determining the deviations from an inverse size dependence that we have previously reported for compound semiconductor materials. Using first-principles density functional theory calculations, we have explored how these factors influence particle stability under a variety of conditions.
\end{abstract}




\section{INTRODUCTION}

The binding or cohesive energy of nanoparticles is of importance because it determines a wide range of physical properties including melting point, congruent vapor pressure, sintering rate and solubility. This is especially true in the case of smaller particles where these properties are often difficult to determine experimentally. Fortunately, first principles theoretical calculations using, for example, density-functional theory can be used to obtain nanoparticle energies with a reasonable degree of accuracy. From these theoretically obtained energies, many relevant physical properties can be estimated to supplement the experimental situation. Furthermore, such calculations can now be performed relatively easily, particularly in the small size range where experimental determinations are most difficult.

The existing literature on the cohesive or binding energy of nanoparticles is primarily based on the assumption that this energy is governed by nearest neighbor interactions. This assumption leads to a linear dependence of this binding energy on the inverse size of the particles (1-4). For many metals, this nearest neighbor assumption is fairly good, and, at least for low aspect ratio particles, a linear dependence of the binding energy on the reciprocal particle radius results. This simple dependence allows for rather simple estimations of various physical properties for both single particles and for ensembles of

particles based solely on the bulk binding energy and the size or size distribution. 
However, for non-metallic materials with covalent or ionic bonding, the assumption that the coherent energy is simply governed by the number of nearest neighbors is no longer valid. This is particularly true for semiconductor nanoparticles where both covalency and ionicity play a major role in the bonding and hence in determining the binding energy of small clusters and nanoparticles as well as of macroscopic, bulk-like constructs. Therefore, in order to better understand the fundamental physical properties of small compound nanoparticles, in general, and of semiconductor nanoparticles in particular, we have undertaken a theoretical study of their cohesive energies as a function of their size and of a variety of other factors that determine their detailed atomistic bonding.

Previously, we have shown that, unlike most metals, the per-atom-pair binding energy of compound semiconductor clusters and small nanoparticles is not a linear function of the inverse of the particle diameter or radius (5). It is, rather, closer to a quadratic dependence, and the per atom-pair binding energy approaches that of the bulk material more rapidly with increasing size than for most metals (5). This behavior is similar to that of the elemental semiconductors, C, Si and Ge, and of the Group IV metals, Sn and $\mathrm{Pb}$. (6). Interestingly enough, it also resembles the non-linear inverse size dependence seen for metal oxides $(7,8)$.

It is the purpose of this work to explore how a variety of factors, including ionicity, the underlying "bulk" structure, surface reconstruction, charge balance and hybridization, strain, stoichiometry and adsorbates, influence the stability of small compound semiconductor clusters and nanoparticles. It should be noted that these systems are 
sufficiently complicated that the task of considering all of the convoluted interactions among these factors would be truly monumental. Therefore, we seek a more modest goal of illustrating how some of these factors operate in specific circumstances with the hope of illuminating the larger problem.

There already exist a wide variety of publications on compound semiconductor clusters and small nanoparticles $(9-37)$. These include experimental studies on CdS nanoparticles by Goldstein, Esher and Alivisatos (9), as well as theoretical calculations on CdS and CdSe clusters by Troparevsky and Chelikowsky (10) and by Deglman, Ahlricks and Tsereteli (11) among others (12-14). ZnSe and $\mathrm{ZnS}$ clusters have also been studied theoretically by Deglman, et al. (11). GaAs clusters have been modeled by various groups including Chelikowsky and coworkers $(15,16)$ and others $(17-25)$. GaN clusters have also been studied by Pandey and coworkers (26-28) as well as others (2932). AlP has also been studied by a number of investigators (33-37). This listing is not exhaustive, but is just meant to give some sense of the breadth of the existing literature in this field. Here, as in our earlier studies, we will attempt to differ from previous efforts by focusing less on the energy variations of different configurations for a given cluster size, and more on the overall behavior as the size of the particle is varied.

\section{MODELING}

First principles, density functional theory (DFT) calculations were used to determine the binding energy, or enthalpy of formation, for the compound semiconductor clusters and small nanoparticles in the generalized gradient approximation (GGA) including spin- 
polarization. The geometry and energetics of the clusters were calculated using the

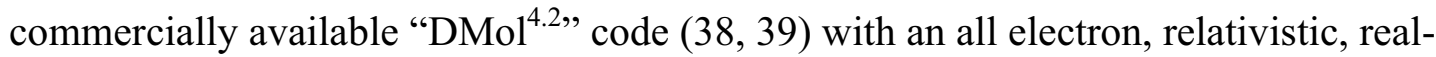
space numerical basis set ("DND"). In this code, the GGA is implemented with the Perdew-Wang-91 (40) parameterization of the correlation energy. Geometric optimization was achieved by allowing the structure to relax until the quantummechanical force on each atom became smaller than $0.05 \mathrm{eV} / \AA$ and the changes in energy and dimension were less than $0.002 \mathrm{eV}$ and $0.005 \AA$, respectively. For the small clusters, several different starting configurations were generally used, and the resulting optimized structures were then examined to determine the most stable configuration. Bulk reference materials were calculated as previously described (5-8).

\section{RESULTS}

To summarize our earlier results, in Figure 1 are shown the normalized, per atom-pair (M-X) binding energies as a function of $1 / \mathrm{n}$ for AlP, CdS, CdSe, GaN, GaAs, ZaS, and ZnSe. In order to show all of the data on the same graph, they are plotted as a function of both reduced energy and reduced reciprocal radius. Normalization of the energy along the $y$-axis was achieved by dividing the particle per atom-pair binding energies by that for the bulk, and referencing that to the energy for a single pair of $\mathrm{M}-\mathrm{X}$ atoms. In addition, we have, in essence, normalized the reciprocal size along the $\mathrm{x}$-axis as well by using $1 / \mathrm{n}$, where $\mathrm{n}$ is the cube root of the number of atom-pairs in the cluster or nanoparticle, as our variable. Here, $n=r / r_{0}$ where $r$ is the effective radius of the particle and $r_{0}$ is the average radius of a single pair of $M-X$ atoms. Noting that, at least for low aspect-ratio particles, $\mathrm{n}$ scales with $\mathrm{r}$, the radius of the particle, this graphic representation 
is equivalent to plotting the per-atom binding energies as a function of the reduced inverse size of the particles. In Figure 1, the point on the far right of the diagram refers to a single atom-pair $(\mathrm{n}=1)$ while the point on the far left refers to the bulk material $(\mathrm{n}=$ $\infty)$

As can be seen, the per atom-pair binding energies deviate from a linear dependence on $1 / \mathrm{n}$ (or $1 / \mathrm{r}$ ) for all of the compound semiconductors studied here. As previously noted, this behavior is in sharp contrast to the linear dependence determined for most metals (5, 6). Beyond that, by plotting the normalized energy, we emphasize the differences between these materials. As can be seen, while AlP and GaAs show an approximately quadratic dependence on 1/n, GaN shows substantially less bowing while $\mathrm{ZnS}$ and $\mathrm{ZnSe}$ show an even greater deviation from a linear $1 / \mathrm{n}$ dependence. In fact, the degree of deviation seems to correlate most strongly with the degree of ionicity; being most marked for the relatively ionic semiconductors (such as $\mathrm{ZnS}$ ) and least apparent for $\mathrm{GaN}$ which, as previously noted, shows "metallic" behavior for several bulk surface structures $(5,41)$.

A second factor that determines the overall binding energy of a cluster or a small nanoparticle is, of course, its detailed atomic structure. This factor is closely tied in with strain and with bonding considerations such as hybridization and dangling orbital occupation. As mentioned in the introduction, there exist a large number of publications on the structure of small compound semiconductor clusters (9-37). While it is not the intent of this paper to review this body of work, it is worthwhile noting that there exist a variety of similarities and differences among the results for different materials. For the 
smallest unit, $\mathrm{M}_{1} \mathrm{X}_{1}$, the solution is trivial and all of the compound semiconductors have the form of a linear diatomic molecule.

However, for the $\mathrm{M}_{2} \mathrm{X}_{2}$ cluster, the results become more interesting. For example, Deglmann, et al. found that for $\mathrm{Zn}_{2} \mathrm{~S}_{2}, \mathrm{Zn}_{2} \mathrm{Se}_{2}, \mathrm{Cd}_{2} \mathrm{~S}_{2}$ and $\mathrm{Cd}_{2} \mathrm{Se}_{2}$, the structure was a rhombus with an $\mathrm{X}-\mathrm{M}-\mathrm{X}$ angle varying between about $111^{\circ}$ for $\mathrm{Cd}_{2} \mathrm{~S}_{2}$ to about $118^{\circ}$ for $\mathrm{Zn}_{2} \mathrm{Se}_{2}$ (11). Troparevsky, et al. found similar results for $\mathrm{Cd}_{2} \mathrm{~S}_{2}$ and $\mathrm{Cd}_{2} \mathrm{Se}_{2}$ (10). In these cases, the bond around the metal angle is less acute than that around the $\mathrm{X}$ atom with the M-M distance being the closest homonuclear distance. In contrast, $\mathrm{Ga}_{2} \mathrm{~N}_{2}$ is also a rhombus, but the situation is reversed with the N-Ga-N angle being most acute, and the N-N distance being the smallest homonuclear distance, reflecting the strength of the N-N bond (26). In fact, this N-N bond strength can destabilize other $\mathrm{Ga}_{\mathrm{m}} \mathrm{N}_{\mathrm{m}}$ clusters that are stable or at least metastable for other compound semiconductor clusters, leading to decomposition with the formation of one or more $\mathrm{N}_{2}$ molecules upon geometric optimization $(5,26)$.

In contrast, for the $\mathrm{M}_{3} \mathrm{X}_{3}$ cluster, a buckled six-membered ring with alternating $\mathrm{M}$ and $\mathrm{X}$ atoms appears to be the most stable configuration not only for $\mathrm{Zn}_{3} \mathrm{~S}_{3}, \mathrm{Zn}_{3} \mathrm{Se}_{3}, \mathrm{Cd}_{3} \mathrm{~S}_{3}$ and $\mathrm{Cd}_{3} \mathrm{Se}_{3}$ but also for $\mathrm{Ga}_{3} \mathrm{~N}_{3}(10,11,28) . \mathrm{Ga}_{3} \mathrm{As}_{3}$ shows a different structure, however. For example, recent work by Kikuchi, et al. using first principles GW calculations, indicate that a less symmetric structure with $\mathrm{C}_{\mathrm{s}}$ symmetry and both Ga-Ga bonds and AsAs bonds is most stable (17). This $\mathrm{Ga}_{3} \mathrm{As}_{3}$ structure is similar to an earlier one proposed by Chelikowsky and coworkers based on DFT calculations (16). Interestingly enough, 
for the $\mathrm{M}_{6} \mathrm{X}_{6}$ clusters, $\mathrm{Ga}_{6} \mathrm{As}_{6}$ is based on a doubled version of the buckled six-membered ring as are the $\mathrm{Zn}$ and $\mathrm{Cd}$ sulfides and selenides $(5,10,11)$. This structure can be envisioned as one ring being placed over another with alternating $\mathrm{M}$ and $\mathrm{X}$ atoms such that $\mathrm{M}-\mathrm{X}$ bonds are formed between the two rings. As an aside, a careful analysis of why As-As and $\mathrm{Ga}-\mathrm{Ga}$ bonds exist in $\mathrm{Ga}_{3} \mathrm{As}_{3}$ but not in $\mathrm{Ga}_{6} \mathrm{As}_{6}$ might shed light on why these homonuclear bonds are found in some of the more complicated GaAs bulk surface reconstructions, but not in others (42).

These brief remarks barely scratch the surface of a comparative study among the compound semiconductor cluster configurations and are merely intended as an indication of the complexities in this area. Obviously, for these small, uncapped, stoichiometric $\mathrm{M}_{\mathrm{m}} \mathrm{X}_{\mathrm{m}}$ clusters, bonding considerations including hybridization, dangling orbital occupation and strain are operative factors. However, before turning to a more detailed discussion of these factors, let us first consider the influence of the underlying structural motif in somewhat larger structures.

Going next to small nanoparticles, with dimensions on the order of a nm or so, the underlying core structure plays a role. Needless to say, when a particle becomes large enough, it is to be expected that the interior of the particle will have the same structure as the most stable bulk modification of the parent material. Conversely, as discussed above, for small clusters there are an insufficient number of atoms to achieve a bulk-like configuration and bonding considerations similar to those that govern conventional 
molecules dominate. Let us next consider what happens in the intermediate range of small nanoparticles with, perhaps, 20-100 atoms.

In attempting to construct $\mathrm{Cd}_{\mathrm{m}} \mathrm{Se}_{\mathrm{m}}$ particles in this size range, Deglmann, et al. (11) found that attempts to base these small nanoparticles on the zinc blende (cubic) structure were not particularly successful. In general, distortions of the molecular geometry occurred to the extent that it was difficult to observe the underlying packing structure, and, in some cases, the clusters even lost $\mathrm{Cd}$ atoms. Conversely, they were successful at building and optimizing a variety of small nanoparticles based on the wurtzite (hexagonal) bulk structure.

We observed similar results for other compound semiconductors. For example, in Figure 2, we show both an $\mathrm{Al}_{25} \mathrm{P}_{25}$ particle based on the zinc blende structure, which is the stable bulk configuration for this material, and an $\mathrm{Al}_{22} \mathrm{P}_{22}$ particle based on the wurtzite structure. (Note that the zinc blende $\mathrm{A} / \mathrm{B} / \mathrm{C}$ repeat pattern along the (111) axis and the wurtzite $\mathrm{A} / \mathrm{B}$ pattern along the $\mathrm{C}$ axis can still be discerned in these clusters.) Calculations for these two structures were done under identical conditions as described in the modeling section. These structures were chosen as the most compact, and hence most stable, in this size range for their relevant bulk basis structures. Even despite the fact that it is smaller, the wurtzite based cluster is more stable, and has a per atom-pair binding energy of about $-7.3 \mathrm{eV}$ relative to that for the zinc blende based structure of $-6.7 \mathrm{eV}$. These energies may be compared with the per atom-pair bulk binding energy of $-8.34 \mathrm{eV}$. 
This preference for a wurtzite-based core architecture underlying the structure of the small nanoparticles in this intermediate region would appear to be based on the fact that this structure is simpler than the zinc blende structure in the sense that it has an $\mathrm{A} / \mathrm{B}$ rather than an $\mathrm{A} / \mathrm{B} / \mathrm{C}$ repeat motif along the direction of the $\mathrm{M}-\mathrm{X}$ bonds. This allows for the construction of small particles with fewer dangling orbitals along the corners and edges. In general, these structures require less rearrangement to reach the closest minimum in free energy. (As a word of caution, the closest minimum, may not be the lowest minimum of course.) For materials where the bulk structure is zinc blende, there obvious must be a particle size where a cross-over occurs from wurtzite to zinc blend as the core or bulk energetics begin to dominate over the surface energetics. Unfortunately, our calculations did not extend to particles large enough for this transition to be observed.

The role that the dangling orbitals play in the stability of clusters and small nanoparticles is the next factor that we will consider. Just as with bulk surface reconstructions, the structure and energetics of these particles are strongly influenced by the dangling orbital occupations. This factor is, of course, highly correlated with charge balance, both bonding and dangling orbital hybridization and the reconstruction of the atoms on the surface of the particle. This factor also influences, and is influenced by, strain within the particle.

Many of the same considerations that govern surface reconstructions are operative in the case of nanoparticles. For the compound semiconductors, in general, the unhybridized $p$ orbitals on the metal atoms tend to be above the Fermi level. Therefore, when a metal 
atom occurs at a surface with one broken bond, rather than retaining the bulk $s p^{3}$ configuration, it prefers to form the remaining three bonding orbitals into a planar, $s p^{2}$ configuration leaving the dangling orbital with a predominantly $p$ character. Therefore, the metal dangling orbital will usually be above the Fermi level and will be empty (43, 44). Similarly, two broken bonds tend to lead to $s p$ bonding with two empty $p$-like dangling orbitals.

Conversely, the Group V or VI atoms tend to have their unhybridized $p$ orbitals below the Fermi level, so that these orbitals will prefer to be filled. Therefore, when these atoms occur at a surface with one broken bond, both the bonding orbitals and the non-bonding orbital will, together, retain a non-planar, three dimensional configuration, that is closer to the bonding arrangement which exists in the bulk than to the arrangement seen for surface metal atoms $(43,44)$.

The interplay among these effects is shown in Figure 3 for a small 48 atom nanoparticle of $\mathrm{Zn}_{24} \mathrm{~S}_{24}$. In Figure 3a, we show the starting structure which has an equal number of $\mathrm{Zn}$ and $\mathrm{S}$ atoms, all with a bulk-like tetrahedral configuration. There are three bilayers of 16 atoms each in the basic wurtzite configuration. Particularly in the top and the bottom layer of this particle, many of the atoms will have one, or even two, broken bonds, and will be forced to rehybridize away from the bulk $s p^{3}$ configuration.

In Figure $3 \mathrm{~b}$ is shown the structure of the $\mathrm{Zn}_{24} \mathrm{~S}_{24}$ unit after geometric optimization. As can be seen, of the eight $\mathrm{Zn}$ atoms in the top layer, the three more central atoms have 
three bonds, and have relaxed into a planar, 3 -fold $s p^{2}$ bonding configuration. They will also have a single, empty non-bonding orbital perpendicular to the top layer. Of the remaining $\mathrm{Zn}$ atoms in this plane, four are along the edge of the particle and one is at a corner. In the unreconstructed bulk configuration, these five $\mathrm{Zn}$ atoms are only 2-fold bonded, and would therefore seek a linear, $s p$ bonding configuration with two empty $p$ like dangling orbitals. (The actual situation is, of course, more complicated than this as the paucity of bonding opportunities forces some additional bonding between this top layer $\mathrm{Zn}$ atoms and the mid layer $\mathrm{S}$ atoms that would not occur in the bulk. After geometric optimization, the $\mathrm{Zn}-\mathrm{S}$ distance between planes has been reduced from $4.01 \AA$ to $2.42 \AA$. This can be compared with the $2.28 \AA$ intra-planar $\mathrm{Zn}-\mathrm{S}$ distance for these corner $\mathrm{Zn}$ atoms.) It can also be seen in Figure $3 \mathrm{~b}$ that the corner 2 -fold $\mathrm{Zn}$ atom is constrained such that it cannot achieve a S-Zn-S angle of $180^{\circ}$. This causes considerable strain in this corner position, a point which we will return to below. While this $\mathrm{Zn}_{24} \mathrm{~S}_{24}$ unit is actually stable, with a $-5.4 \mathrm{eV}$ per atom-pair bonding energy, the rehybridization of the surface, edge and corner atoms causes a significant distortion of the atomic geometry away from that of the parent wurtzite structure.

While this distortion can be accommodated in the $\mathrm{Zn}_{24} \mathrm{~S}_{24}$ particle, in a similar, wurtzitebased $\mathrm{Cd}_{22} \mathrm{~S}_{22}$ unit shown in Fig. 4a, it causes sufficient strain that the particle is inherently unstable. This inherent instability is illustrated in Fig. $4 \mathrm{~b}$ where it can be seen that, upon geometric optimization, the particle appears to deconstruct explosively. Here, the starting structure of the $\mathrm{Cd}_{22} \mathrm{~S}_{22}$ unit, is a 44 atom thin platelet of two bilayers having 10 atoms of one kind and 12 of the second kind in each bilayer. This behavior is similar 
to that seen by Deglmann, et al. where some zinc selenide based CdSe particles actually ejected $\mathrm{Cd}$ atoms upon geometric optimization (11).

Several points are worth noting here. The first is that both the $\mathrm{Zn}_{24} \mathrm{~S}_{24}$ and the $\mathrm{Cd}_{22} \mathrm{~S}_{22}$ particles have an equal number of sulfur and metal dangling orbitals. Therefore, the electron population that is emptied out from the metal dangling orbitals can be accommodated in the sulfur dangling orbitals. Thus, electron counting considerations are obeyed and strain is indicated as the destabilizing factor.

This point is reinforced by the observation that the next larger particle in this series, the $\mathrm{Cd}_{32} \mathrm{~S}_{34}$ unit with three bilayers is, in fact, stable with a $-5.09 \mathrm{eV}$ binding energy per atom-pair. As will be discussed below for the case of $\mathrm{Cd}_{19} \operatorname{In}_{3} \mathrm{~S}_{22}$, partially filled cation dangling orbitals can stabilize these particles by allowing the corner cations to buckle out of the plane, thereby reducing the strain. Therefore, in the $\mathrm{Cd}_{32} \mathrm{~S}_{34}$ case, it is probable that the extra charge generated by the surplus of $\mathrm{S}$ atoms stabilizes this particle. The additional observation that, unlike the $\mathrm{Cd}_{22} \mathrm{~S}_{22}$ particle, the $\mathrm{Zn}_{22} \mathrm{~S}_{22}$ particle is stable, with a $-5.74 \mathrm{eV}$ per atom-pair binding energy also reinforces this point when it is considered that the $\mathrm{Zn}$ atoms are smaller in size than the $\mathrm{Cd}$ atoms. This allows the doubly bonded $\mathrm{Zn}$ atoms to approach a linear bonding configuration with more ease than the larger $\mathrm{Cd}$ atoms are allowed within the constraints of the S bonding energetic and geometric requirements. 
Even at this relatively small size of only 44 atoms, this is a tremendously complex problem that involves many trade-offs among the bond angles and lengths to achieve the lowest free energy situation. However, taking a number of these factors together, including the fact that the corner, two-folded metal atoms appear to be particularly strained, suggest that stability might be achieved by reducing the strain on the corner metal atoms by relaxing the requirement that they strive for a linear, $s p$ bonding configuration. There are several ways to approach this problem.

One possible way to reduce the strain on the corner metal atoms is by substituting a Group III metal atom, such as Ga or In, with three valance electrons, for the corner $\mathrm{Cd}$ atoms. As In is to the right of $\mathrm{Cd}$ in the periodic table it is more electronegative, and therefore, will have lower lying valence orbitals. Consequently, these Group III atoms will have lower-lying dangling orbitals. As all of the $\mathrm{S}$ dangling orbitals are filled, the additional electrons will partially occupy the In dangling orbitals. This will move their optimal geometry for two-fold bonded species away from the linear $s p$ configuration toward a non-linear $s p^{2}$ configuration, which should allow these metal atoms to move upward out of the plane, thereby reducing the strain in the corners.

In Figure 5, we show what actually happens when three In atoms are substituted for the corner $\mathrm{Cd}$ atoms. In Figure 5a is shown the unrelaxed $\mathrm{Cd}_{19} \mathrm{In}_{3} \mathrm{~S}_{22}$ structure, and in Figure $5 \mathrm{~b}$ is shown the same particle after geometric optimization. As can be seen, the particle does not fall apart, but forms a stable, reconstructed unit that has a per-atom binding energy of $-5.12 \mathrm{eV}$. As can also be seen, the In atoms are displaced out of the plane with 
a non-linear bonding configuration. Thus, as conjectured above, substituting three In atoms into corner positions in the $\mathrm{Cd}_{22} \mathrm{~S}_{22}$ unit does actually stabilize the particle. This is despite the fact that the In atoms are marginally larger than the $\mathrm{Cd}$ atoms.

As might be expected, substituting three smaller Ga atoms, instead of In atoms, also produces a stable particle with a slightly more stable per atom-pair binding energy of $5.19 \mathrm{eV}$, though part of this difference may be attributable to the difference between Ga-S and In-S bonding energies. Given the success of this strain relief mechanism it might be interesting to try to adsorb three $\mathrm{H}$ atoms on the corner $\mathrm{Cd}$ atoms in $\mathrm{Cd}_{22} \mathrm{~S}_{22}$ in order to see if stability could be achieved via this mechanism. Additionally the adsorption of three $\mathrm{H}$ atoms on three $\mathrm{S}$ atoms might also force electrons into the corner $\mathrm{Cd}$ dangling orbitals. However, these and related additional calculations are properly the subject of a separate paper.

The results shown in Figure 5 are one example of the effects of substituting one species for another on the per atom-pair binding energy of clusters and small nanoparticles. Just as with bulk compound semiconductors, there are a vast number of permutations that can be considered. In this work, we have studied only a very small subset of them. In our first example, we have substituted a single $\mathrm{Zn}$ atom into four different positions on the top layer of a $\mathrm{Cd}_{32} \mathrm{~S}_{34}$ particle. As one might expect, all of these substitutions produce stable configurations with small changes in the average per atom-pair binding energy that vary somewhat depending upon the position where the substitution was made. Because of the somewhat stronger $\mathrm{Zn}-\mathrm{S}$ bond, the average energy is slightly greater than that for 
$\mathrm{Cd}_{32} \mathrm{~S}_{32}(-5.14 \mathrm{eV})$. Perhaps more interesting is the fact that substituting a $\mathrm{Zn}$ atom into an edge position (Figure 6a) produces greater stabilization than substitution into the corner position (Figure 6b). This difference between bulk and particle distribution may simply reflect the fact that the latter has more degrees of freedom for relieving strain, or it may be a by product of the uneven M:X stoichiometry. Further calculations on stoichiometric particles are needed to answer this question.

Rather more surprising, at least initially, is what happens when we substitute a number of $\mathrm{Zn}$ atoms into a $\mathrm{Cd}_{32} \mathrm{~S}_{32}$ cluster (Figure 7). Here, distributing the $\mathrm{Zn}$ atoms over the three bilayers creates a more stable situation than concentrating them on one or two bilayers. This is unlike what occurs in a bulk structure containing two different types of metal atoms where the different species prefer to segregate onto the same plane ((111) in zinc blende and (0001) in wurtzite) for strain relief (45).

In our discussion of strain relief in $\mathrm{Cd}_{22} \mathrm{~S}_{22}$ particles, we conjecture that the adsorption of three $\mathrm{H}$ atoms on the particle might stabilize it. The use of adsorbates on nanoparticle systems has been employed for a variety of other purposes, as well. Alivisatos and coworkers have used adsorbates to control growth of nanoparticles and to keep them suspended in solution such that they can be manipulated for a variety of purposes. They have "capped" CdS and CdSe nanoparticles with a variety of adsorbates including functionalized alkane chains (46), polyphosphates (47), tetrabutyl ammonium (47), thioglycolate (48), and thiophenol (48). 
In one study (9), they used temperature dependent electron diffraction to measure the melting points of CdS nanoparticles ranging in size from about $1.3 \mathrm{~nm}$ to $4 \mathrm{~nm}$. They observed a large depression in the melting temperature with decreasing size ranging down to over $50 \%$ for sufficiently small nanoparticles. A comparison of their experimental results with our theoretical calculations provides an interesting example of several other effects of adsorbates on nanostructure stability in compound semiconductors.

Based on our model calculations, we can assume that the per atom-pair binding energy, $\mathrm{E}_{\mathrm{nb}}$, has the approximate form

$$
\mathrm{E}_{\mathrm{nb}}=\mathrm{E}_{\mathrm{b}}^{\infty}\left(1-1 / \mathrm{n}^{2}\right)
$$

where $\mathrm{E}_{\mathrm{b}}{ }^{\infty}$ is the binding energy for the bulk material for the bulk material and $\mathrm{n}$ is the cube root of the total number of MX atom-pairs in the particle $(5,6)$. Noting that the bulk melting point of most compound semiconductors is proportional to their binding energy to a good approximation (5), and making a similar assumption for the corresponding nanoparticles, we can write

$$
\mathrm{T}_{\mathrm{nm}}=\mathrm{T}_{\mathrm{m}}^{\infty}\left(1-1 / \mathrm{n}^{2}\right)
$$

or, equivalently, 


$$
\mathrm{T}_{\mathrm{nm}}=\mathrm{T}_{\mathrm{m}}^{\infty}\left[1-\left(\mathrm{r}_{\mathrm{o}} / \mathrm{r}_{\mathrm{n}}\right)^{2}\right]
$$

where $r_{o}$ is the average radius of an $n=1$ particle and $r_{n}=n r_{o}$. Here $T_{n m}$ is the melting point of a low aspect-ratio nanoparticle with $\mathrm{n}^{3} \mathrm{MX}$ atom pairs and $\mathrm{T}_{\mathrm{m}}{ }^{\infty}$ is the bulk melting point.

For CdS, taking $\mathrm{T}_{\mathrm{nm}} / \mathrm{T}_{\mathrm{m}}{ }^{\infty}=\mathrm{E}_{\mathrm{nb}} / \mathrm{E}_{\mathrm{b}}{ }^{\infty}, \mathrm{T}_{\mathrm{m}}{ }^{\infty}=1750^{\circ} \mathrm{C}$ or $2023 \mathrm{~K}$ and $\mathrm{E}_{\mathrm{b}}^{\infty}=-4.35 \mathrm{eV}$, we can plot the CdS data in Figure 1 as a function of $1 / r^{2}$ in Figure 8 . Here $r_{o}$ was taken as $2.279 \AA$. As can be seen, our data are well fit by a straight line in this size range justifying our assumption of an approximate $1 / \mathrm{n}^{2}$ or $1 / \mathrm{r}^{2}$ dependence.

For comparison, we have taken the data of Alivisatos and coworkers on the capped CdS nanoparticle melting points and re-plotted it as a function of $1 / \mathrm{r}^{2}$ in Figure 9. Here, despite some scatter in the experimental data, we also find a plausible $1 / \mathrm{r}^{2}$ dependence. There are, however, two significant differences between Figures 8 and 9. One is that the apparent bulk melting points are not the same. Assuming that the experimental temperature measurements are correct, not a trivial matter in an electron microscope when radiation losses become significant, it can be seen that the extrapolated "bulk" value for the capped nanoparticles is only about $2 / 3$ the value that we have assumed for pure bulk CdS. This may be taken as an indication that the adsorbate or cap lowers the overall binding energies of the nanoparticles. 
The other significant difference between Figures 8 and 9 is that the slope is different between the two plots. Even though the scales of the two figures are different, it can easily be seen that the slope is substantially steeper for the experimental data than for the calculated data. One qualitative explanation for this difference can be found in noting that the experimental data is best fit by an $r_{o}$ value of just under $10 \mathrm{~A}^{\circ}$, rather than the 2.279 $\mathrm{A}^{\circ}$ value assumed for the calculated plot. This difference in $r_{o}$ is taken as an indication that the adsorbate or capping "fluffs up" the nanoparticles, giving them substantially larger radii than the corresponding uncapped CdS nanoparticles.

\section{SUMMARY}

In summary, using first-principles density functional theory calculations, we have explored a variety of factors that influence the stability of compound semiconductor cluster and small nanoparticle binding energies. These include ionicity, underlying structure, hybridization and dangling orbital occupation, strain, stoichiometry and the presence of adsorbates. Greater ionicity appears to lead to proportionally greater binding energy, and relatively ionic materials approach their bulk binding energies most rapidly with increasing particle size. For small clusters, bonding considerations and structure are inexorably entwined and, on a somewhat larger scale, for small nanoparticles, a wurtzitetype underlying structure is more favorable than a zinc blende-type structure as the underlying core architecture in the cases studied here. Hybridization, dangling orbital occupation and strain all are closely interdependent and are crucial in determining the stability of both clusters and nanoparticles. 
Both substitutional atoms and adsorbates have the capability to either stabilize or destabilize the particles, depending upon the circumstances. A comparison between experimental and calculated melting points for CdS nanoparticles has also been made and suggests some of the effects introduced by capping these particles. Future work comparing experiment and theory, not only for melting temperatures, but also for other quantities that are dependent on binding energies, such as nanoparticle sublimation, sintering and solubility, is needed and should further illuminate the effect of these and other factors. Finally, studies of the various factors that shape clusters and nanoparticles are of significance not only for themselves, but also for what new insights they can bring to the studies of how these factors can influence the structures of bulk surfaces and interfaces. The converse is true as well as these problems are strongly correlated.

\section{ACKNOWLEDGEMENT}

This work was supported by the Division of Material Sciences and Engineering of the Office of Basic Energy Sciences, through the DOE Idaho Operations Office Contract DEAC07-05ID14517. The author would also like to thank Chris Palmstrøm and Phyllis King for their kind assistance in this work.

\section{REFERENCES}


1. See, for example, S. C. Vanithakumari, and K. K. Nanda, J. Phys. Chem. B 110, 1033 (2006), and references therein.

2. See, for example, W. H. Qi, M. P. Wang, M. Zhou, and W. Y. Hu, J. Phys. D: Appl. Phys. 38, 1429 (2005), and references therein.

3. See, for example, Chang Q. Sun, H. L. Bai, S. Li, B. K. Tay, C. Li, T. P. Chen, and E. Y. Jiang, J. Phys. Chem. B 108, 2162 (2004), and references therein.

4. See, for example, M. Wautelet, J. P. Dauchot, and M. Hecq, J. Phys.: Condens. Matter 15, 3651 (2003), and references therein.

5. H. H. Farrell, C. D. Van Siclen, D. M. Ginosar, L. M. Petkovic, and R. D. Parra, submitted to J. Vac. Sci. Technol. B.

6. H. H. Farrell and C. D. Van Siclen, J. Vac. Sci. Technol. B, 25, 1441 (2007).

7. R. D. Parra and H. H. Farrell, to be published.

8. R. D. Parra and H. H. Farrell, to be published.

9. A. N. Goldstein, C. M. Esher, and A. P. Alivisatos, Science 256, 1425 (1992).

10. M. C. Troparevsky and J. R. Chelikowsky, J. Chem. Phys. 114, 943 (2001).

11. P. Deglmann, R. Ahlrichs, and K. Tsereteli, J. Chem. Phys. 116, 1585 (2002).

12. B. Li, L. Wang, Q. Jin, Z. Guo, S. Tang, and D. Ding, J. Porous Mat. 9, 287 (2002).

13. V. S. Gurin, Colloid. Surface. A, 202, 215 (2002).

14. S.-Y. Ren and S.-F. Ren, J. Phys. Chem. Solids 59, 1327 (1998).

15. N. Binggeli and J. R. Chelikowsky, Appl. Phys. Lett. 66, 1316 (1995).

16. I. Vasiliev, S. Öğüt, and J. R. Chelikowsky, Phys. Rev. B 60, R8477 (1999).

17. E. Kikuchi, S. Iwata, S. Ishii, and K. Ohno, Phys. Rev. B 76, 075325 (2007).

18. P. Karamanis, D. Bégué, and C. Pouchan, J. Chem. Phys. 127, 094706 (2007). 
19. P. Piquini, S. Canuto, and A. Fazzio, Nanostruct. Mater. 10, 635 (1998).

20. X. Zhu, Spectrochim. Acta, Part A 61, 2730 (2005).

21. K. M. Song, A. K. Ray, and P. K. Khowash, J. Phys. B 27, 1637 (1994).

22. W. Zhao, P. L. Cao, B. X. Li, B. Song, and H. Nakamatsu, Phys. Rev. B 62, 17138 (2000).

23. J. J. BelBruno, Heteroat. Chem. 14, 189 (2003).

24. L. Lou, L. Wang, L. P. Chibante, R. T. Laaksonen, and P. Nordlander, J. Chem. Phys. 94, 8015 (1991).

25. M. A. Al-Laham and K. Raghavachari, J. Chem. Phys. 98, 1 (1993).

26. A. K. Kandalem, R. Pandey, M. A. Blanco, A. Costales, and J. M. Recio, J. Phys. Chem. B 104, 4361 (2000).

27. A. K. Kandalem, M. A. Blanco, and R. Pandey, J. Phys. Chem. B 105, 6080 (2001) and ibid. 106, 1945 (2002).

28. P. P. Korambath and S. P. Karna, J. Phys. Chem. 104, 4801 (2000).

29. B. Song and P. L. Cao, J. Chem. Phys. 123, 144312 (2005).

30. J. J. BelBruno, Heteroat. Chem. 11, 281 (2000).

31. B. H. Boo and Z. Y. Liu, J. Phys. Chem. A 103, 1250 (1999).

32. A. Costales and R. Pandey, J. Phys. Chem. A 107, 191 (2003).

33. C. R. Zhang, H. S. Chen, Y. H. Chen, W. J. Feng, and G. J. Wu, Chin. J. Inorg. Chem. 22, 1038 (2006).

34. A. Costales, A. K. Kandalam, R. Franco, and R. Pandey, J. Phys. Chem. B 106, 1940 (2002).

35. M. Driess, S. Kuntz, C. Monse, K. Merz, Chem. Eur. J. 6, 4343 (2000). 
36. A. Tomasulo and M. V. Ramakrishna, Z. Phys. D-Atoms Mol. Clusters 40, 483 (1997).

37. M. A. Allaham, G. W. Trucks, and K. Raghavachari, J. Chem. Phys. 96, 1137 (1992).

38. B. Delley, J. Chem. Phys. 92, 508 (1990).

39. B. Delley, J. Chem. Phys. 113, 7756 (2000).

40. J. P. Perdew and Y. Wang, Phys. Rev. B 45, 13244 (1992).

41. See, for example, J. E. Northrup and J. Neugebauer, Phys. Rev. B 53, 10477 (1996), and references therein.

42. See, for example, H. H. Farrell, J. Lu, B. D. Schultz, A. B. Denison, and C. J.

Palmstrøm, J. Vac. Sci. Technol. B 19, 1597 (2001).

43. See, for example, H. H. Farrell and C. J. Palmstrøm, J. Vac. Sci. Technol. B, 8, 903 (1990).

44. See, for example, H. H. Farrell, M. C. Tamargo, S. M. Shibli and Y. Chang, J. Vac.

Sci. Technol. B, 8, 884 (1990).

45. See, for example, B. A. Philips, I. Kamiya, K. Hingerl, L. T. Florez, D. E. Aspnes, S. Mahajan, and J. P. Harbison, Phys. Rev. Lett., 74, 3640 (1995).

46. J. G. Tobin, V. L. Colvin, and A. P. Alivisatos, J. Vac. Sci. Technol. A 9, 852 (1991).

47. V. L. Colvin, A. P. Alivisatos, and J. G. Tobin, Phys. Rev. Lett. 66, 2786 (1991).

48. M. Haas and A. P. Alivisatos, J. Phys. Chem. 96, 6756 (1992). 


\section{FIGURE CAPTIONS}

Fig. 1; The normalized binding, or cohesive, energy for several compound semiconductors, is shown as a function of $1 / \mathrm{n}$ where of the cube root of the number of atoms in a particle. The point at the far right is for an MX atom pair, and that are the far left for the bulk material. Normalization was performed by dividing the individual per atom-pair energies by that for the bulk.

Fig. 2; For small nanoparticles, wurtzite-like structures appear to be more stable than those based on the zinc-blende structure. (a) $\mathrm{An}_{\mathrm{Al}_{25}} \mathrm{P}_{25}$ particle based on the zinc blende structure. $\mathrm{Al}$ (dark pink), $\mathrm{P}$ atoms (lavender). Binding energy is about $-6.7 \mathrm{eV}$ per AlP unit. (b) $\mathrm{An} \mathrm{Al}_{22} \mathrm{P}_{22}$ particle based on the wurtzite structure. Binding energy is about $-7.3 \mathrm{eV}$ per AlP unit.

Fig. 3; Just as with bulk surface reconstructions, cluster and nanoparticle structures are strongly influenced by the dangling orbital occupation. In general, metal dangling orbitals tend to be empty, and chalcogenide dangling orbitals tend to be filled. (a) A $\mathrm{Zn}_{24} \mathrm{~S}_{24}$ particle before optimization shown in plan view, all angles tetrahedral. $\mathrm{S}$ (yellow), Zn (blue grey). (b) The same structure after geometric optimization. Note that 2-fold $\mathrm{Zn}$ atoms tend to be $s p$ bonded ( linear), while 3-fold $\mathrm{Zn}$ atoms tend to be $s p^{2}$ bonded ( planar). Conversely, 2 -fold and 3-fold $\mathrm{S}$ atoms have filled dangling orbitals, and therefore more acute angles. 
Fig. 4; Corners and edges introduce strain effects not present on bulk surfaces. An extreme example of strain occurs when we try to make thin platelets of $\mathrm{Cd}_{22} \mathrm{~S}_{22}$. (a) Before optimization. Plan view, left; side view, right. Cd (orange), S (yellow). (b) After optimization. The strain introduced when the corner $\mathrm{Cd}$ atoms attempt to form linear, $s p$ bonds while accommodating the bonding changes in the adjacent $\mathrm{S}$ atoms causes the cluster to "explode" upon geometric optimization.

Fig. 5; The strain seen in Fig. 4 can be relieved by inserting corner atoms that require approximately $s p^{2}$ rather than sp bonding, such as In or Ga. (a) $\mathrm{A} \mathrm{Cd}_{19} \operatorname{In}_{3} \mathrm{~S}_{22}$ structure before optimization. Plan view, left; side view, right. In atoms, (green). (b) The same structure after optimization. Even though In is slightly larger than Cd, substituting In atoms for the corner $\mathrm{Cd}$ atoms changes the bonding requirements and allows the formation of a stable cluster (as do Ga atoms).

Fig. 6; Substituting one $\mathrm{Zn}$ atom for a $\mathrm{Cd}$ atom in a stable $\mathrm{Cd}_{32} \mathrm{~S}_{34}$ cluster produces a small change in the average binding energy per CdS unit. (a) Top left, $\Delta \mathrm{E}_{\mathrm{b}}=-5.170 \mathrm{eV}$. (b) Top right, $\Delta \mathrm{E}_{\mathrm{b}}=-5.162 \mathrm{eV}$. (c) Bottom left, $\Delta \mathrm{E}_{\mathrm{b}}=-5.161 \mathrm{eV}$. (d) Bottom right, $\Delta \mathrm{E}_{\mathrm{b}}$ $=-5.149 \mathrm{eV}$. Because of the somewhat stronger $\mathrm{Zn}-\mathrm{S}$ bonds, the average energy is slightly greater than that for $\mathrm{Cd}_{32} \mathrm{~S}_{34}(-5.09 \mathrm{eV})$, but varies with the position of the $\mathrm{Zn}$ atom.

Fig. 7; Substituting a larger number of $\mathrm{Zn}$ atoms into $\mathrm{Cd}_{32} \mathrm{~S}_{32}$ clusters produced a somewhat more surprising result. For $\mathrm{Cd}_{20} \mathrm{Zn}_{12} \mathrm{~S}_{32}$ clusters, the configuration where the 
$12 \mathrm{Zn}$ atoms are distributed among all three layers (top left) is more stable than that where they are all arranged in the middle layer (top right), or on the outer layers (bottom left and right). (a) Top left, $\Delta \mathrm{E}_{\mathrm{b}}=-5.231 \mathrm{eV}$. (b) Top right, $\Delta \mathrm{E}_{\mathrm{b}}=-5.190 \mathrm{eV}$. (c) Bottom left, $\Delta \mathrm{E}_{\mathrm{b}}=-5.188 \mathrm{eV}$. (d) Bottom right, $\Delta \mathrm{E}_{\mathrm{b}}=-5.153 \mathrm{eV}$. Note that unlike MM'X bulk structures where a given type of metal atom tends to align on (111) planes with other atoms of the same kind, in these clusters, the most stable configurations appear to be those where atoms of a given type are not all arranged on the same plane.

Fig. 8; The effect of adsorbing a "cap" on the surface of a cluster or nanoparticle can be studied in part by comparing the calculated melting point of CdS clusters with the experimental data of Alivisatos, et al. (9). Here, for uncapped CdS particles, the melting point was assumed to be proportional to the binding energy of the particle, and the bulk melting point (far left point) was taken as $2023 \mathrm{~K}\left(1750^{\circ} \mathrm{C}\right)$. The nominal radius of each particle was taken as $r_{0}=2.279 \AA$.

Fig. 9; Experimental data for capped CdS nanoparticles has been obtained by Alivisatos, and coworkers (9). They have shown that the melting point of these CdS nanoparticles decreases with decreasing size (9). Here, the experimental melting points are plotted as a function of $1 / \mathrm{r}^{2}$, and they appear to have a quadratic, rather than a linear, dependence on $1 / \mathrm{r}$ or $1 / \mathrm{n}$. Note that the melting point for the adsorbate- coated nanoparticles appears to be lower and the slope is steeper than for the calculated data in Fig. 8 . 


\section{FIGURES}

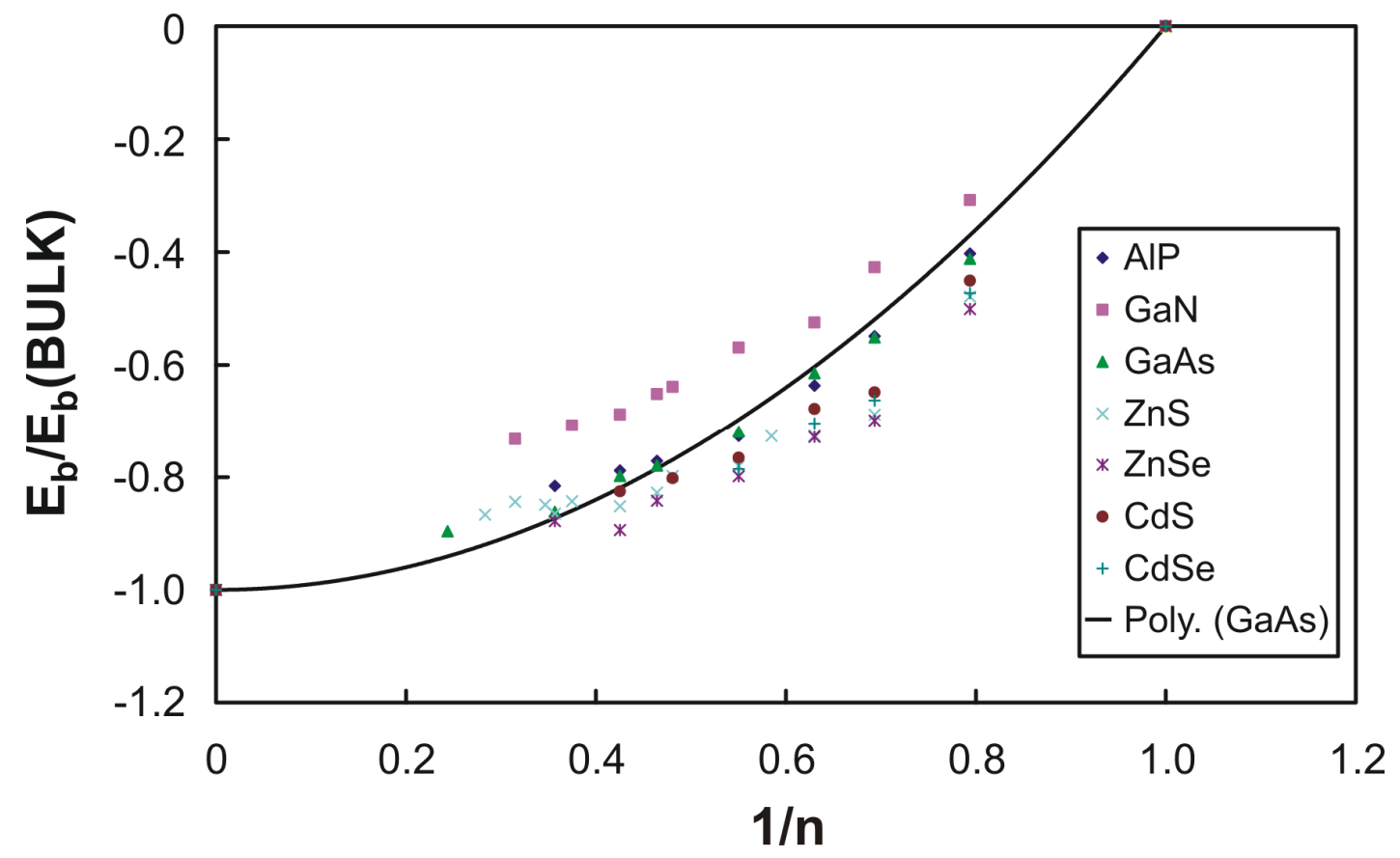

Figure 1. 


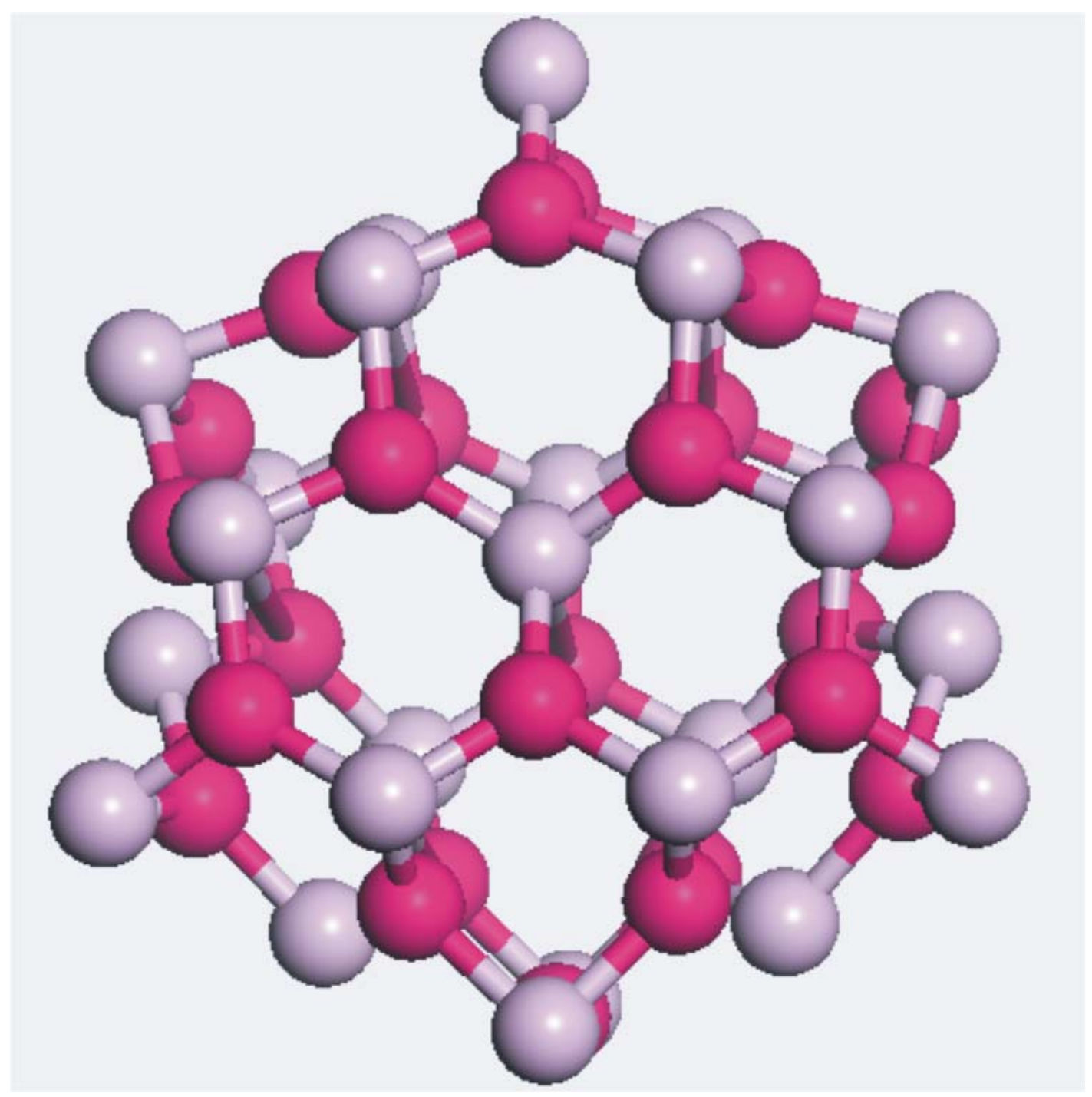

Figure 2a. 


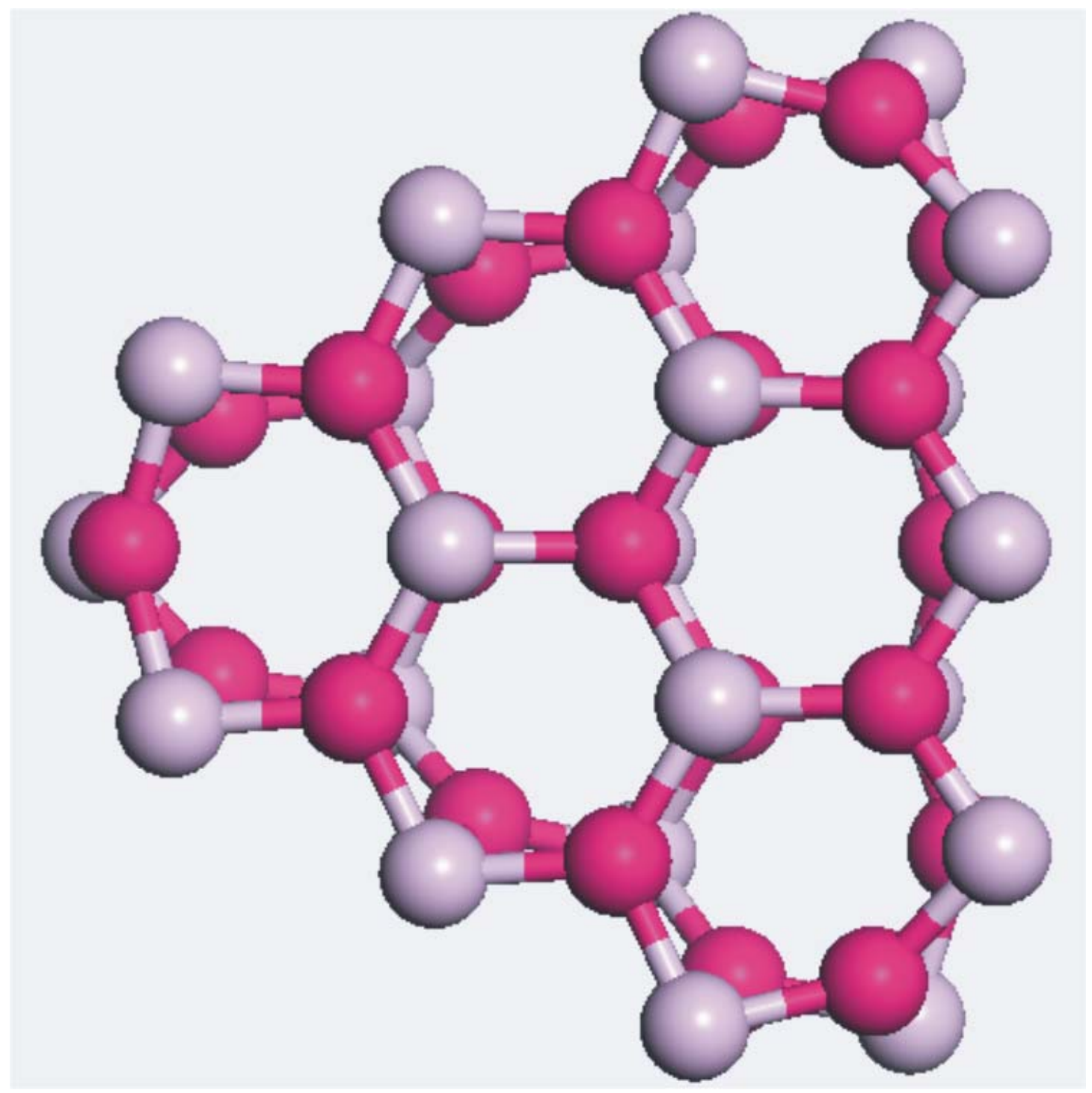

Figure 2b. 


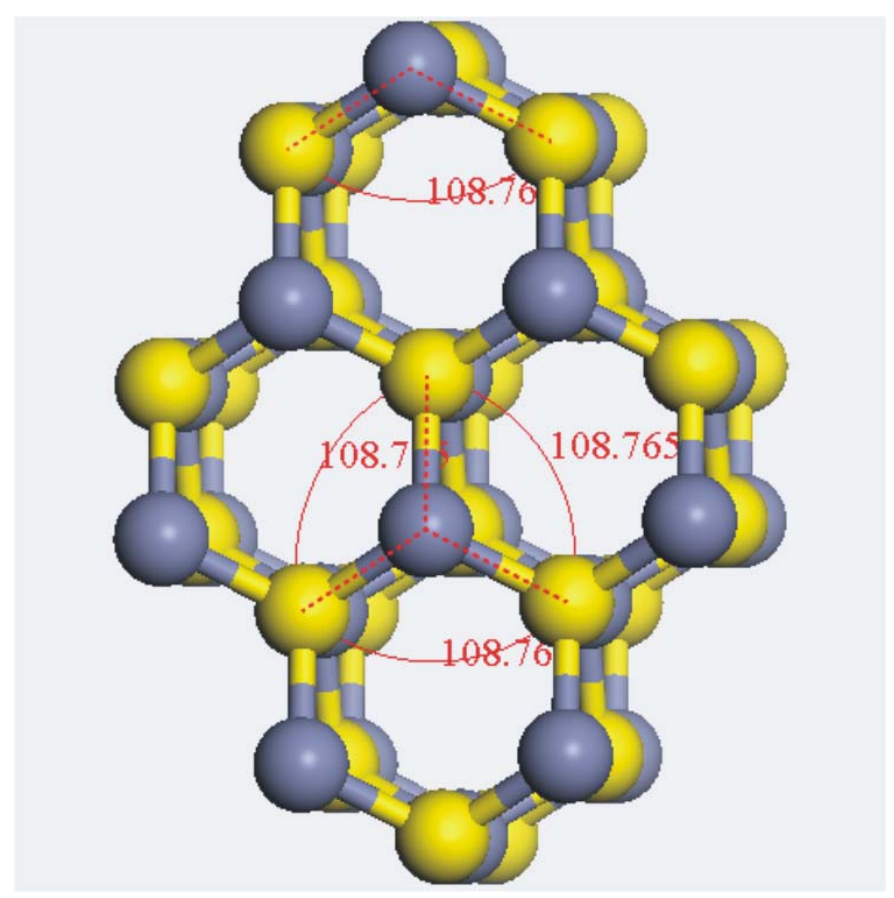

Figure 3a.

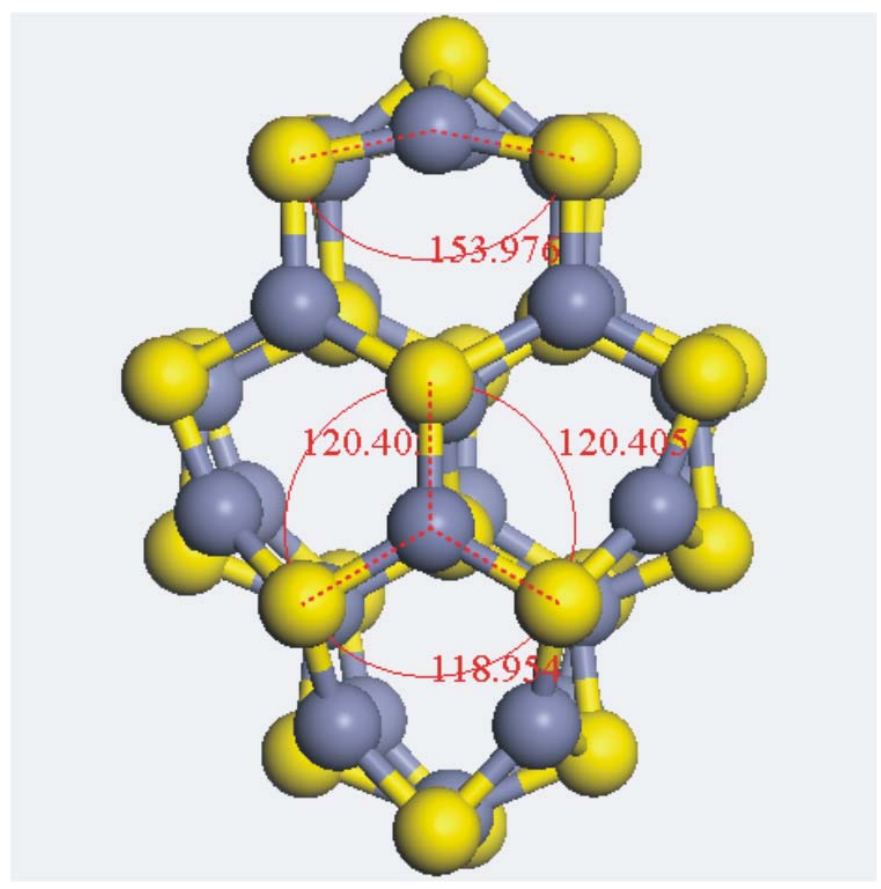

Figure 3b. 

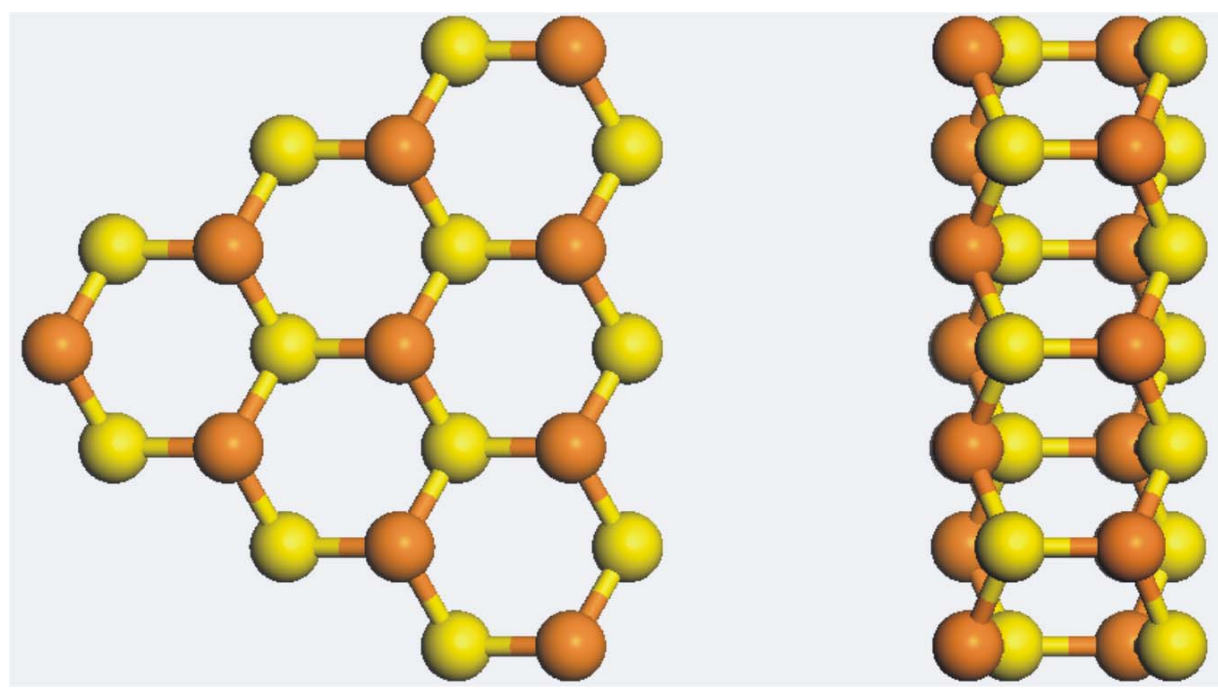

Figure 4a.

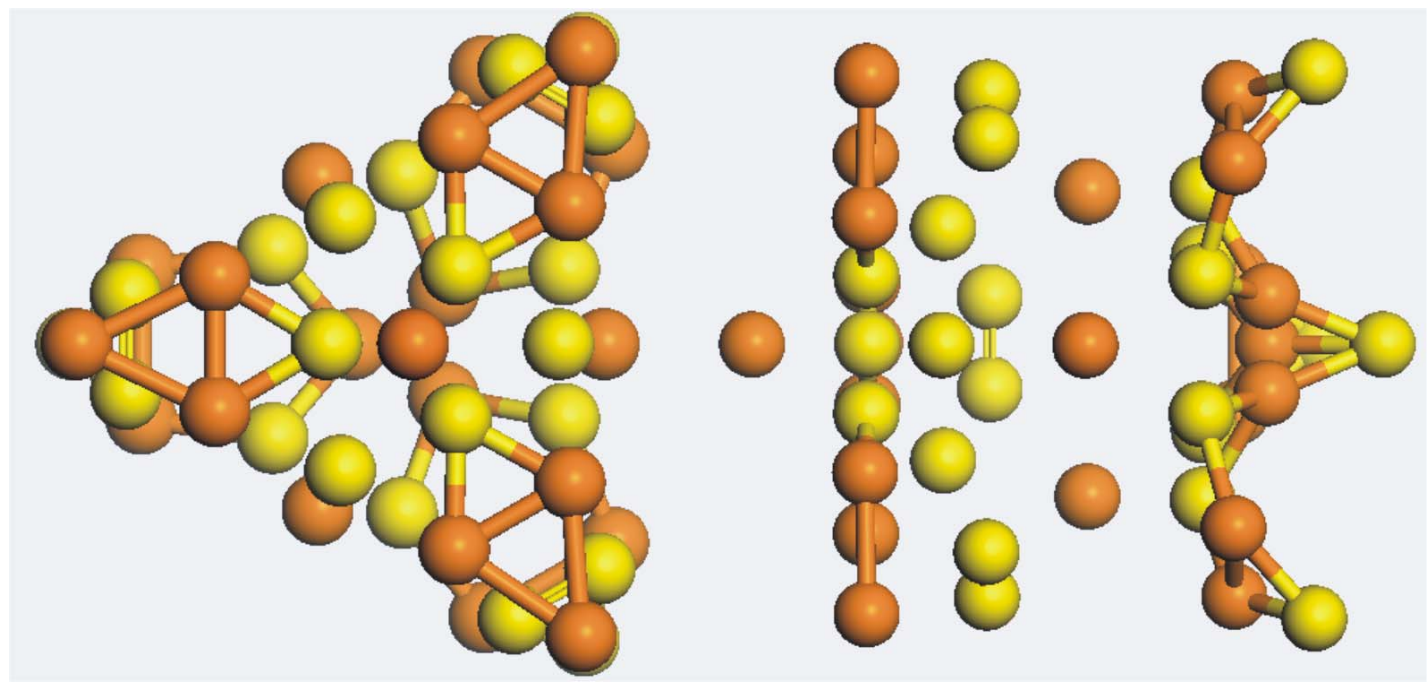

Figure 4b. 


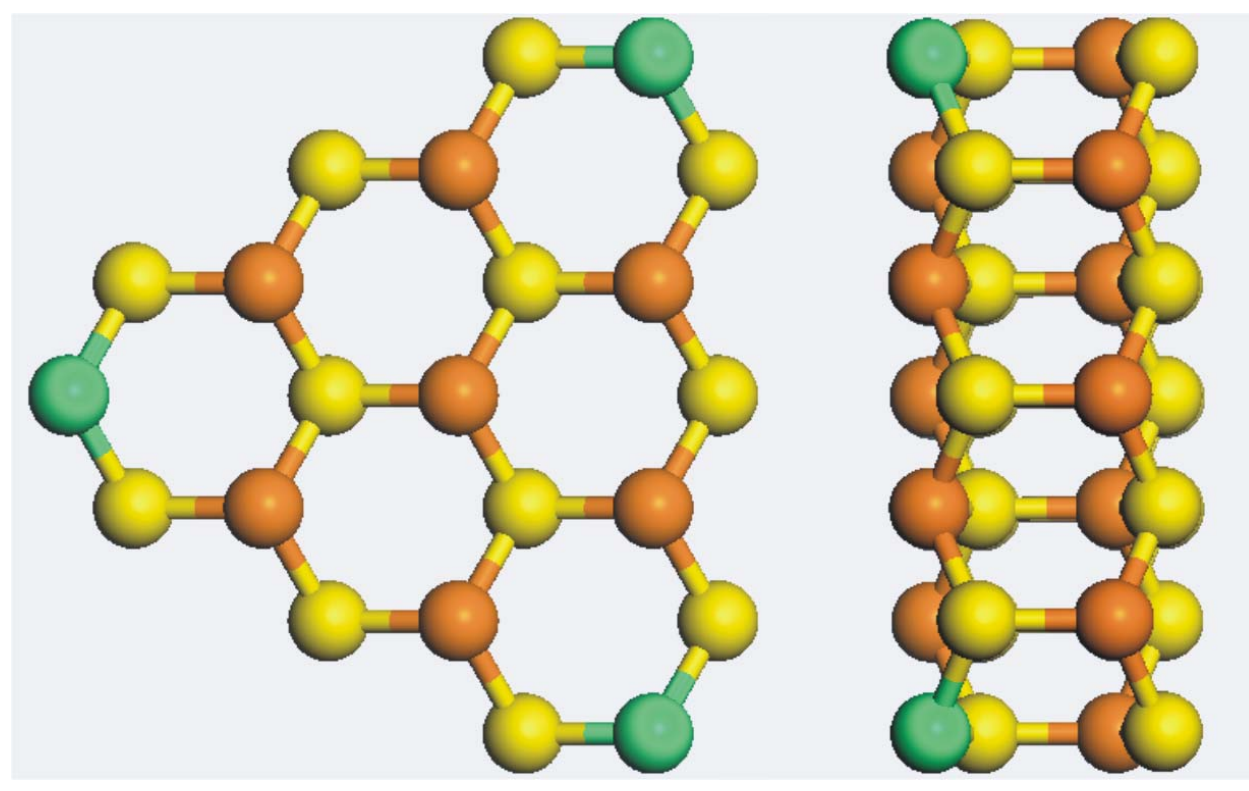

Figure 5a.

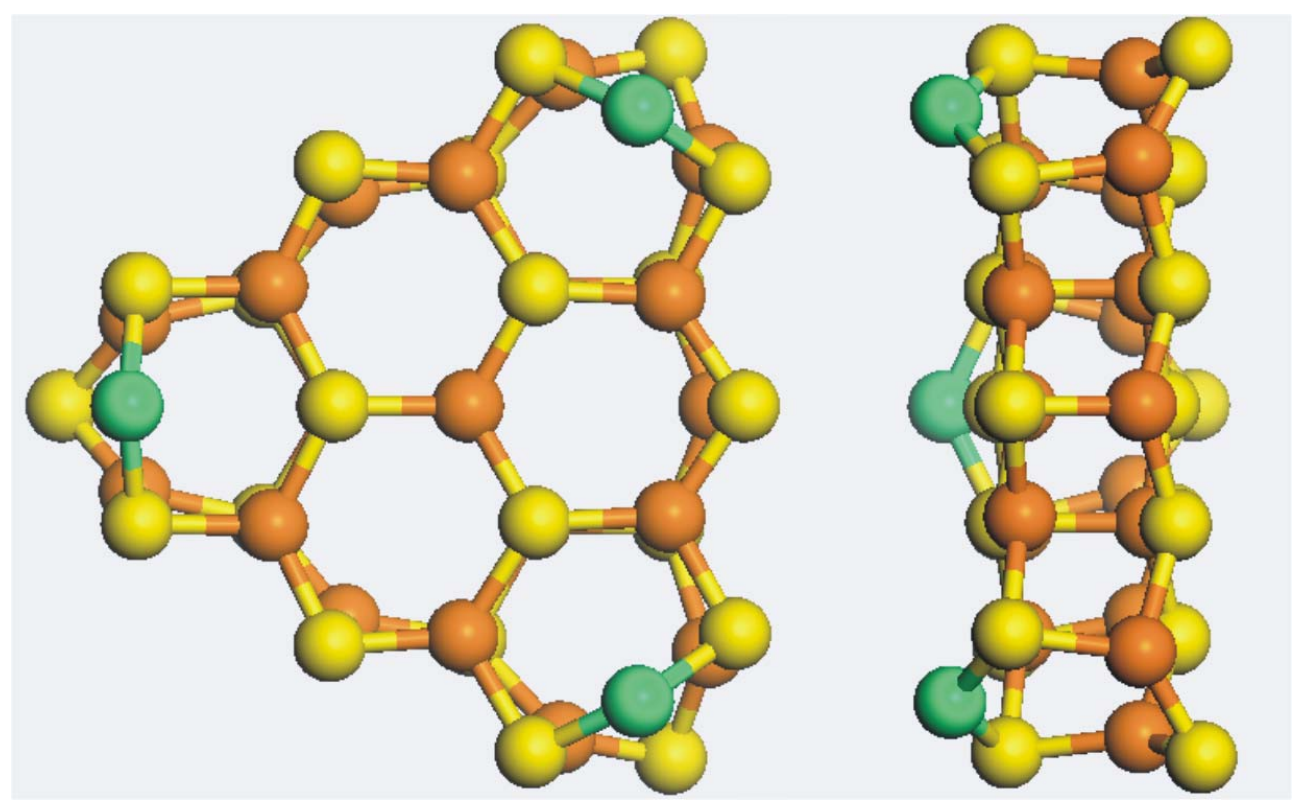

Figure 5b. 


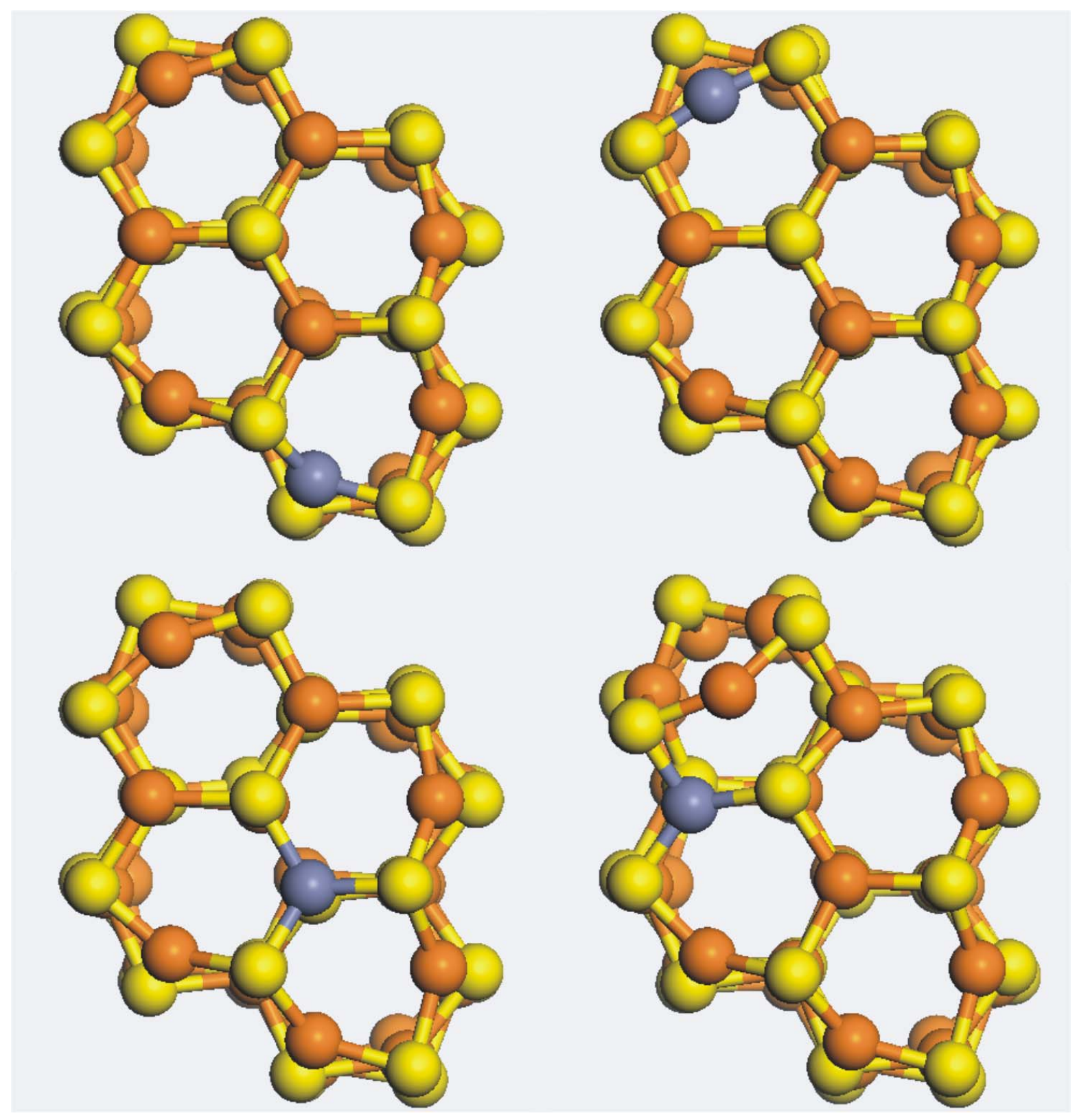

Figure 6. 


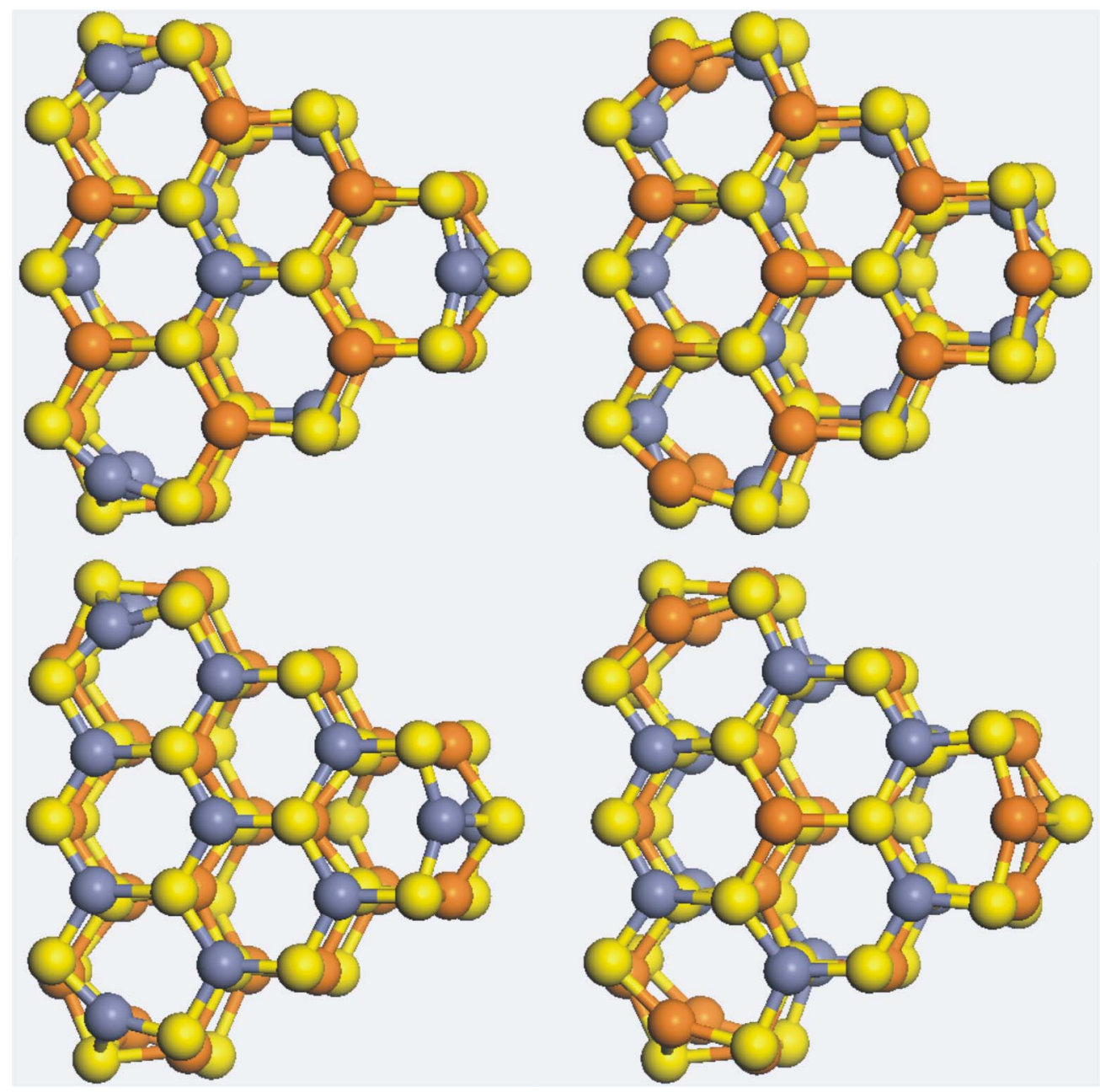

Figure 7. 


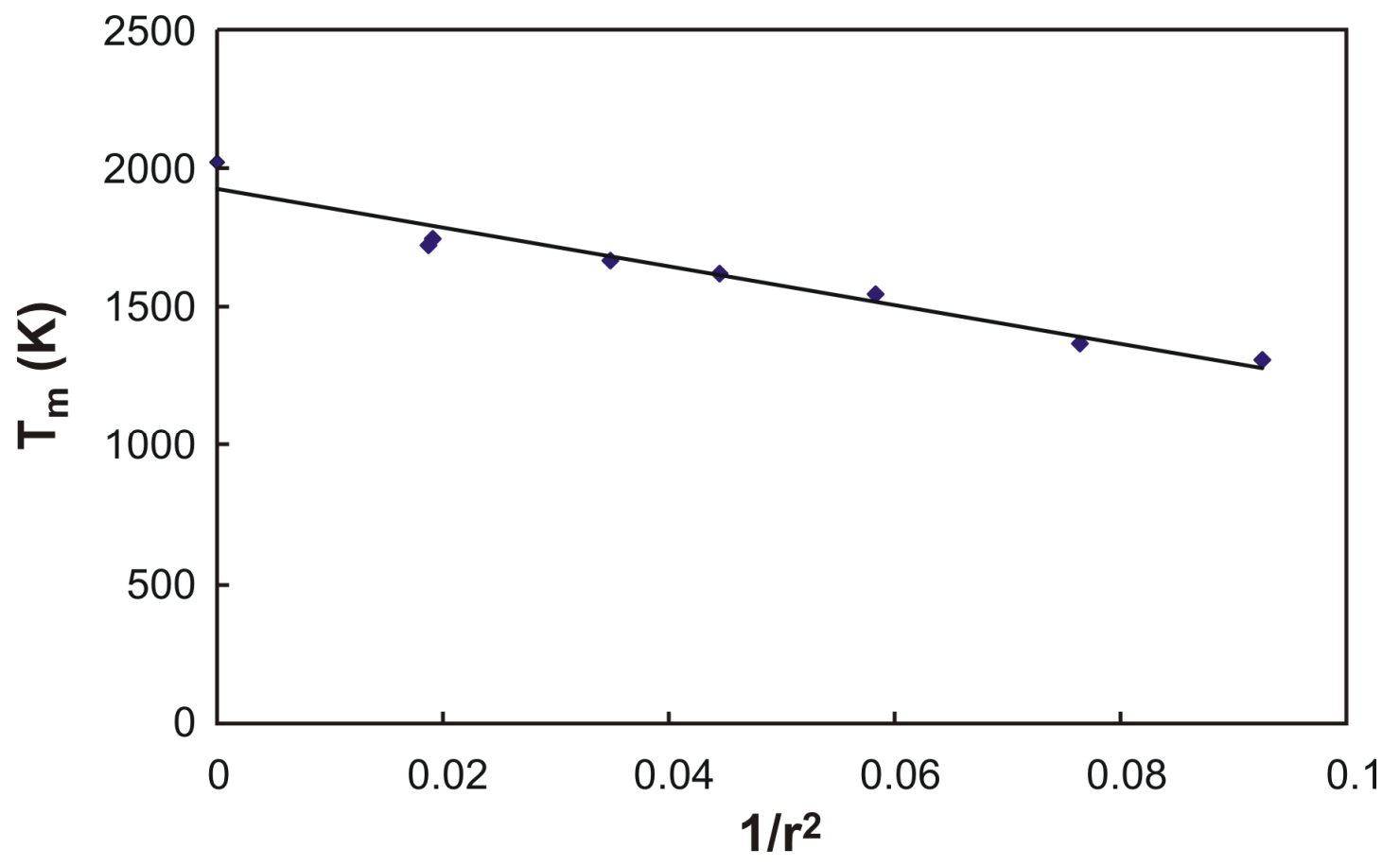

Figure 8. 


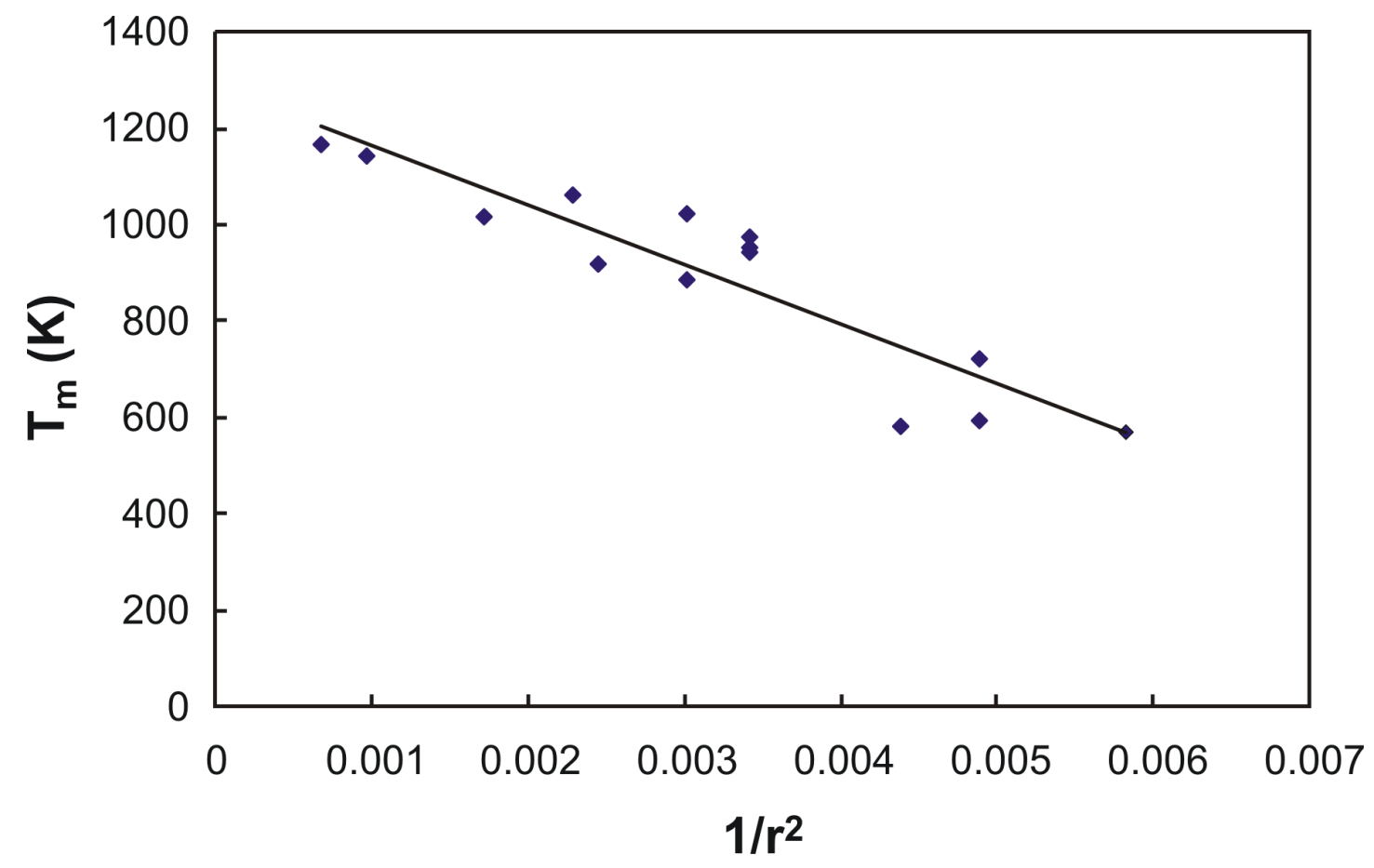

Figure 9. 